\title{
Quand le nom massif et I ou comptable change de référent : recatégorisation, décryptage métonymique et faits de polysémie lexicale
}

\author{
El Mustapha Lemghari ${ }^{1, a}$ \\ ${ }^{1}$ Université Cadi Ayyad - Marrakech, Maroc
}

\begin{abstract}
Résumé. La distinction massif/comptable a actuellement le vent en poupe. Ce renouveau d'intérêt peut s'expliquer aussi bien par l'éclairage qu'elle apporte à la relation entre langage, pensée et réalité (cf. Chierchia, 2010) que par la complexité de la structure informative des noms massifs et comptables eux-mêmes, ainsi que de leur corrélation. Cette communication entend relancer le débat sur la complexité de cette corrélation à travers l'examen du phénomène de la recatégorisation dans son articulation au phénomène de la polysémie lexicale. Par ailleurs, excepté quelques contributions (Numberg et Zaenen (1997); Kleiber (1999) et Lemghari (2010)), le rapport de la recatégorisation à la polysémie a peu retenu l'attention des linguistes. Dans la littérature sur le sujet, la manifestation d'un nom [+massif] et/ou [+comptable] sous le trait inverse se traduit généralement par une recatégorisation discursive. Du coup, les faits de recatégorisations ne sont pas nettement démêlés des faits de polysémies lexicales. En examinant quelques recatégorisations de type métonymique, nous tenterons d'éclaircir le rôle du phénomène de recatégorisation dans l'enrichissement et la complexification des bases conceptuelles des noms massifs et comptables. Chemin faisant, c'est la conception classique d'une opposition massif/comptable tranchée qui se trouvera remise en cause.
\end{abstract}

\begin{abstract}
Referential change of mass and count nouns: metonymic transfer and lexical polysemy. Much attention is paid nowadays to the mass/count Distinction. Such a particular interest is due to the pervasive role the mass/count Distinction plays in clarifying the relationship between language, thought and reality (Chierchia, 2010), as well as to the conceptual complexity structuring mass and count nouns at large. Expanding on this complexity, this paper intends to tackle mainly the nature of mass and count nouns transfers at the light of the phenomenon of polysemy. Except some studies (Numberg \& Zaenen (1997); Kleiber (1999); Lemghari (2010)), little effort has been devoted to connecting the
\end{abstract}

a El Mustapha Lemghari : e.lemghari@uca.ma 
mass/count Distinction to the phenomenon of lexical polysemy. It is even commonly assumed in the classical literature that the occurrence of a noun basically either [+mass] or [+count] under the converse trait ends up in a discursive transfer process. Accordingly, cases of discursive transfers are not drastically distinguished from those of lexical polysemy. Our purpose is to deal with metonymical transfers. We will attempt to highlight their role in enriching and complexifying the conceptual bases of mass and count nouns. Expectedly, it is the radical mass/count Distinction that will be called into question.

\section{Introduction}

Le phénomène de la distinction massif / comptable a actuellement le vent en poupe. Ce renouveau d'intérêt pour la problématique est alimenté dans une large mesure par deux paradigmes de recherches. D’un côté, bon nombre de spécialistes pensent que ce phénomène est susceptible d'éclaircir la relation qui unit le langage à la pensée et au monde extérieur. Ce paradigme de recherches est principalement cognitiviste en ce qu'il s'attèle à rendre compte de la nature des systèmes conceptuels (G. Lakoff, 1987 ; M. Johnson, 1987 ; L. Talmy, 2000, entre autres). Bon nombre des travaux menés dans cette perspective sont d'orientation comparative, et tâchent d'apporter un élément de réponse à la question de l'universalité de la distinction massif / comptable (L. Cheng et R. Sybesma, 1999 ; J. Chierchia, 1998 et 2010 ; C. Goddard, 1994 et 2009 ; C. Goddard et A. Wierzbicka, 2002 ; A. Wierzbicka, 1988, entre autres). Ce paradigme comprend également des recherches psychologiques et psycholinguistiques (P. Bloom et D. Keleman, 1995 ; M. Imai et D. Gentner, 1997 ; D. Barner et J. Snedeker, 2005 ; entre autres). D'autre part, d'autres chercheurs (la liste est très longue ici) privilégient la perspective strictement linguistique (au sens large du terme). Ce paradigme tend surtout à revisiter la distinction traditionnelle massif / comptable à la lumière de nouveaux problèmes ou la redécouverte de problèmes anciens. Toujours est-il qu'il reste fondamentalement tourné vers le langage. L'objet d'étude commun aux recherches effectuées dans ce sens est de rendre compte de la manière dont chaque grammaire encode la distinction massif / comptable. Le débat dans ce paradigme est d'autant plus animé qu'on est venu à constater que cette distinction est très complexe et qu'elle s'articule à d'autres faits de langage. En règle générale, la complexité du phénomène est due au fait que les frontières entre les massifs et les comptables ne sont pas aussi nettes que le laissait entendre la grammaire traditionnelle. Autrement dit, en dehors des noms massifs et des noms comptables canoniques, il existe des noms qu'on ne saurait aisément ranger dans l'une ou l'autre catégorie ; c'est le cas des noms du type de pain, herbe, linge, etc. D'autres noms, tels que mobilier, quincaillerie, etc., ont un statut ambigu qui déjoue toute la batterie de tests habituellement utilisés pour distinguer franchement entre les deux catégories.

La présente étude se réclame du deuxième paradigme. Nous entendons en effet prolonger le débat sur la complexité de la distinction massif / comptable de par l'examen du phénomène de la recatégorisation dans sa relation au phénomène de la polysémie lexicale. Nous visons donc à atteindre deux objectifs.

- Après avoir procédé à des comptes rendus critiques de quelques propositions émises dans le cadre général de la recatégorisation, nous postulerons deux modes de recatégorisation, qui sont assez répandus dans l'usage du français, mais qui demeurent tout à fait méconnus. Ces modes se manifestent dans les énoncés figuratifs qui comportent des noms massifs et / ou comptables à décryptage métonymique. 
- nous tenterons de placer la polysémie au cœur du débat sur le chapitre des noms massifs et des noms comptables. L'objectif sera de mettre en relation le phénomène de la recatégorisation et celui de la polysémie lexicale. L'accent sera mis sur leur recoupement et leur bifurcation.

Cette étude est d'inspiration cognitive; elle s'inscrit surtout dans le cadre de la Grammaire Cognitive de R.W. Langacker (1987 et 1991). Nous exploiterons, pour l'analyse proposée, notamment les principes et les notions théoriques à la base de la définition de la catégorie nominale, tels que matrice / base conceptuelle, domaine / espace d'instanciation, domaines cognitifs, etc.

\section{Quelques modes de recatégorisation comptable $\rightarrow$ massif}

\subsection{Le broyeur universel}

Le broyeur universel est postulé par J. Pelletier (1975). C’est une machine fictive destinée à broyer, donc à réduire en bouillie massive, les objets comptables de départ. Ainsi, si on fait passer un chêne, objet comptable, dans le broyeur, on obtient à la sortie une bouillie qu'on ne peut plus désigner comme un chêne, mais plutôt comme du chêne.

Pratiquement, le broyeur permet la massification des objets comptables ; son effet est donc matériellement concevable. Mais, son défaut majeur procède de sa puissance elle-même : il n'existe pas en effet d'objet qui puisse y passer et ne pas se transformer en bouillie massive. La thèse du broyeur prête le flanc à la critique à volonté (cf. M. Galmiche, 1989). Pourtant, on lui reconnait un avantage crucial, qui réside dans la distinction entre deux types de broyeur : le broyeur qualitatif et le broyeur quantitatif. Le broyeur qualitatif se vérifie davantage dans le domaine des noms propres métonymiques, comme le fait remarquer $\mathrm{G}$. Kleiber (1994 : 108). Il permet de répondre à la nécessité de reconnaître les productions artistiques sous le nom de l'artiste lui-même :

\section{(1) a. Cette femme aime porter du Courrèges \\ b. J'aime écouter du Bach, du Chopin, etc.}

Le broyeur quantitatif, quant à lui, est à l'œuvre dans les emplois où les entités se trouvent matériellement broyées :

(2) Dans cette tarte, il y a de la pomme. (M. Galmiche, 1989 : 75)

A nos yeux, cette distinction, invoquée comme argument à l'appui du bien-fondé du broyeur, n'est pas invincible. En ce qui concerne le broyage quantitatif, on voit mal en effet comment du Courrèges, $d u$ Bach et $d u$ Chopin peuvent subir un certain broyage de quelque sorte qu'il soit. Dans ces exemples, les référents des noms massifiés sont abstraits. Ceci est, bien entendu, contradictoire avec le propre d'une machine broyeuse. A son tour, le broyeur quantitatif ne passe la rampe que dans certains cas, comme dans (2). Et même dans ce cas, l'état broyé de la pomme n'est pas nécessairement le seul choix possible. Par exemple, dans (3), de la pomme de terre dénote un référent ambigu : il peut s’agir en effet de pommes de terre broyées ou de pommes de terre coupées en petits morceaux ou en tranches :

\section{(3) Dans cette soupe, il y a de la pomme de terre}

Par ailleurs, nous semble-t-il, le broyage altère la sémiosis des objets sur lesquels il opère. En effet, dans la mesure où le broyeur transforme tout objet comptable en massif, il est présupposé que le rapport du total signifiant / signifié au référent demeure inchangeable avant et après le broyage. Les données réelles prouvent le contraire cependant : une chaise broyée est-elle vraiment de la chaise ? Parallèlement, une voiture passée dans le broyeur ressemblerait plutôt à de la ferraille qu'à de la voiture. Parmi les nombreuses critiques que M. Galmiche (1989 : 67) a adressées à la thèse du broyeur, nous retenons celle-ci, qui porte 
sur la sémiosis des objets : « Toute entité structurellement composite, dont la solidarité des parties repose sur l'unité de l'ensemble résiste à l'épreuve du broyeur ». Il est clair que Galmiche fait référence ici aux objets composites du gendre de chaise, table, voiture, etc. Mais, pour y regarder de plus près, même les objets naturels, dès lors qu'ils sont broyés, perdent, pour la majorité, le lien sémiotique qui les associe à leurs signes respectifs. Dans ce sens, la bouillie massive qui résulte du broyage d'un ou de plusieurs chênes désigne un autre référent qu'on ne saurait adéquatement designer par l'expression partitive $d u$ chêne. Qui plus est, dans des énoncés comme (4), les expressions massives du chêne et $d u$ peuplier sont rétives à des interprétations en termes de référence à des réalités broyées de chêne et de peuplier respectivement :

\section{(4) Il y a beaucoup de chêne et de peuplier par-là ${ }^{1}$}

Tout compte fait, le broyeur modifie totalement la structure des objets broyés, engendrant ainsi des réalités qui ont souvent dans la langue un statut sémiotique autonome. Ainsi le broyage d'un chêne ou d'un peuplier ou de tout autre arbre livrera la même bouillie massive, qu'on dénomme habituellement bois déchiqueté ou bois granulé. Chose plus curieuse encore, dans l'usage courant, on réfère souvent au bois du chêne, de l'’acajou, etc., en somme, au bois des arbres utilisés en ébénisterie et en marquèterie, par les expressions massives correspondantes : du chêne, de l'acajou, etc. Il est bien évident que les référents de pareilles expressions ne sont pas saisis comme étant broyés. Un problème se soulève alors: s'agit-il ici d'une recatégorisation comptable $\rightarrow$ massif ou d'une polysémie lexicale ? (Nous reviendrons plus en détail sur ce phénomène un peu plus loin).

Pour conclure ce point, on notera que le champ d'application du broyeur est très restreint. De plus, son caractère artificiel le rend moins compatible avec les données naturelles du langage.

\subsection{Le multiplicateur}

Conscient des limites de la thèse du broyeur, M. Galmiche (1989) propose le multiplicateur pour rendre compte de la conversion comptable $\rightarrow$ massif. Soit les exemples suivants :

(5) a. Il y a de la gifle dans l'air

b. Il va y avoir de la chute

c. Le train ne pouvait être à l'heure; Il y a eu de l'arrêt continuellement

d. Il y avait beaucoup de journalistes, il a fallu subir de l'éclair de flash toute la soirée. (M. Galmiche, 1989 : 70)

Les expressions de la gifle, de la chute, de l'arrêt et de l'éclair de flash sont des massifications des noms comptables correspondants. Ces massifications se justifient en termes de la multiplication indéfinie des actions décrites ; d'où l'idée du multiplicateur. Ainsi, (5a) implique que plusieurs gifles ont été échangées, (5b) qu'un ou plusieurs coureurs vont tomber lors d'une course par exemple, (5c) que le train s'est arrêté plusieurs fois, et enfin (5d) que l'éclair de flash s'est produit infiniment. En conséquence, ces énoncés sont identiques en ce qu'ils décrivent les termes comptables recatégorisés en massifs comme désignant des actions sujettes à plusieurs répétitions.

Comme le souligne Galmiche lui-même (1989 : 70), le multiplicateur a pour avantage de rendre compte de certains termes qui résistent à l'épreuve du broyeur, comme il ressort de (6a-b)

(6) Ce week-end, on a fait du kilomètre

(7) Cet écrivain est ennuyeux; il fait du paragraphe de la ligne 
Nous noterons tout de même que ces emplois sont assez forcés, et ce fait explique dans une large mesure pourquoi ils ne sont pas très fréquents dans l'usage courant. Cependant, l'idée de multiplication est bien naturelle dans d'autres emplois tels que les suivants :

(8) Il doit y avoir du canard en quantité ! disait le substitut (...) en observant les terrains d'alentour (...) (Simenon, cité par M. Herslund, 1998 : 70)

(9) Il y a de la truite et du saumon dans cette rivière

En somme, contrairement au broyeur, le multiplicateur semble bien compatible avec les données de la langue. A cet égard, il est possible de massifier plus au moins naturellement tout objet comptable se présentant en quantité indéfinie. Mais très paradoxalement, certains recatégorisations paraissent moins naturelles que d'autres. C'est le cas par exemple de (10) en regard à (8-9) :

(10) Sur la principale, il y avait de la voiture à perte de vue. (M. Galmiche, ibid.)

D’un autre côté, tout comme le broyeur, le champ d'application du multiplicateur est très réduit. Par exemple, il ne permet pas d'élucider les emplois massifs suivants :

(11) Ce boucher vend un peu tout, du bouf, du poulet, du porc et même du sanglier

Quoi qu'il en soit, le broyeur et le multiplicateur tous ensemble n'épuisent pas les modes de massification du comptable ; les données de la langue requièrent en effet l'examen d'autres modes d'explication.

\section{Recatégorisation du massif en comptable}

Notre propos se limite dans cette section à l'examen de quelques modes de recatégorisation qui sous-tendent le passage inverse massif $\rightarrow$ comptable.

\subsection{Le trieur universel}

Sur le modèle du broyeur universel, H. Bunt (1985) propose le trieur universel pour rendre compte de la conversion massif $\rightarrow$ comptable. Cette machine sert en quelque sorte à découper à l'intérieur des substances massives des variétés qualitativement distinctes. Considérons, à titre illustratif, les phrases suivantes :

(12) Je bus deux ou trois coups d'un vin délicieux

(13) En général, je prépare mes tartines avec trois beurres

(14) Les eaux minérales ne sont pas forcément des eaux de table

(15) Elle m'aime d'un amour qui m'étouffe

Vin, beurre, eau et amour sont sous-catégorisés dans la langue en noms massifs. Néanmoins, dans ces exemples, ils sont recatégorisés en comptables. Cette recatégorisation s'explique en termes de la référence à des espèces ou à des sous-espèces.

L’idée du trieur universel ne semble emporter la persuasion d'aucun spécialiste de la distinction massif / comptable. On lui reproche d'un commun accord le fait que la référence aux espèces ou aux sous-espèces ne se solde pas par le changement du trait massif des noms concernés, car, comme le résume $M$. Galmiche (1989 : 73) : « ce sont les espèces qui sont comptées, pas la substance elle-même ».

\subsection{La thèse du conditionneur}

La thèse du conditionneur (Universal packager) est aussi célèbre que celle du broyeur. On en attribue la paternité à David Lewis ${ }^{2}$. A ma connaissance, la meilleure application de 
cette notion aux données du français est celle de M. Galmiche (1989), que nous suivons de très près ici. Pour aller vite, $M$. Galmiche fait remarquer que le français fournit un type de recatégorisation massif $\rightarrow$ comptable qui n'implique d'aucune façon une quelconque référence aux espèces ou aux sous-espèces, comme le montrent les exemples suivants :

\section{(16) a. Un blanc sec \\ b. Deux bières \\ c. Une choucroute garnie}

Les substances massives bière, blanc et choucroute sont versées dans la catégorie comptable en termes de leur conditionnement individualisant et partant, comptable, du type de verre, canette, plat, etc.

M. Galmiche invoque à l'appui de la recatégorisation massif $\rightarrow$ comptable en termes du conditionneur l'argument de l'absence de tout phénomène d'ellipse dans les expressions comptabilisées. En d'autres termes, selon lui, dans les énoncés (16a-c), aucun élément, du type de verres (pour a. et b.) et d'assiette (pour c.) n'est effacé. La preuve en est que « le déterminant - un qui est porteur de la marque du genre grammatical - s'accorde régulièrement avec le nom de la substance ». On constate clairement ce fait dans les énoncés (17) :

\section{(17) a. Un Ricard \\ b. Un coca}

S’il y avait ellipse dans ces énoncés, c'est-à-dire si on référait au nom effacé bouteille, c’est une qui devrait apparaitre à la place de un et non l'inverse.

Il est regrettable que $\mathrm{M}$. Galmiche n'ait pas examiné cet argument à fond ; il aurait fourni la preuve irréfutable de l'un des rares cas où le massif concret se trouve comptabilisé en français. Il parait que M. Galmiche hésite à trancher la question de la recatégorisation des substances massives en comptables en termes du conditionneur, car il constate - mais il ne le dit pas expressément - qu'il y a toujours un certain flottement en matière des emplois où il n'y a pas ellipse et les emplois où il y en a. En effet, quoi qu'il soit convaincu qu' « un Ricard n'est certainement pas « un verre de Ricard » (...), et [qu'] un double Ricard n'est pas, non plus, un «double verre » ", il admet tout de même que les noms recatégorisés vin et eau sous (18a-b) n’ont pas les mêmes dénotations :

\section{(18) a. Vous avez un vin et une eau... \\ b. Et vous me rendez une vin et une eau}

Selon Galmiche, « alors que dans [18] a., il s’agit d’une « unité de vin » (conditionnée dans une bouteille), dans [18] b., il s'agit de la bouteille elle-même, ou plutôt - selon le témoignage de l'informatrice - de la consigne ».

Cet aspect important de la recatégorisation massif $\rightarrow$ comptable dépasse le cadre du présent travail. Nous noterons tout de même que le phénomène de la lexicalisation offre dans ce sens une piste de recherche prometteuse. Aussi est-il plausible de considérer les massifs qui sont conditionnés en comptables sans aucun phénomène d'ellipse comme des expressions lexicalisées et, donc, fixées en tant que telles dans l'usage courant. Ces emplois peuvent résulter en effet de la subjectivation des conditionneurs qui sont à leur origine. Par subjectivation nous entendons, suivant en cela Langacker (1987 et 1991), l'extension sémantique en termes de laquelle une entité originairement objective reçoit une interprétation subjective. Il arrive en effet que les substances massives sujettes à un genre de conditionnement fréquent se figent dans l'usage sous les formes typiques de leurs conditionnements. Il semble clair que dans de tels cas, la conception du conditionneur en tant que contenant physique devient si schématique qu'on ne l'identifie pas immédiatement sous les emplois concernés, comme le montrent (19a-b) :

(19) a. Il met habituellement deux sucres dans son café 


\section{b. Il boit chaque matin un petit Ricard avant de se rendre au travail}

Le processus de subjectivation peut expliquer bien des emplois qu'on a l'habitude de mettre sur le compte de la recatégorisation. L’idée d'un conditionneur est très schématique dans ces exemples: l'expression deux sucres n'évoque que schématiquement l'unité morceau, puisqu'elle ne met pas en profil la forme conditionnante morceau mais la quantité qui est l'équivalent de deux morceaux réunis ${ }^{3}$. Pareillement, l'expression un petit Ricard évoque schématiquement l'unité conditionnante verre, mais c'est à une certaine quantité de Ricard qu'elle réfère. Si tel n’était pas le cas, un énoncé comme (20a) serait inacceptable, comme le montre (20b) :

(20) a. Je veux un petit Ricard dans un verre à ballon

b. ?? Je veux un petit verre de Ricard dans un verre à ballon

Par ailleurs, le conditionneur présente à son tour quelques limites qui se tiennent pour l'essentiel dans son incompatibilité avec les noms massifs abstraits. En effet, dans la mesure où les massifs abstraits sont des « substances abstraites » (cf. Langacker, 1987), ils rechignent à la mise en conditionnement dans des entités individualisantes. Cependant, leur apparition sur la dimension [+comptable] n'est point chose impossible, comme il ressort de (20a-b) :

(21) a. Il ne commettait que violences et cruautés (A. France, Pt Pierre)

b. La paix n'est rien, qu'il faut acheter au prix d'une injustice (Péguy, Argent)

Il est bien clair que les noms violences, cruautés et injustice n’impliquent pas de référence aux espèces ou aux sous-espèces. Ils ne présupposent pas, non plus, un certain conditionnement dans des entités individualisantes. Pourtant, ils apparaissent sous l'aspect du comptable, comme en témoigne leur distribution ave les marqueurs externes de comptabilité (marquage au pluriel (ex. 21a) et compatibilité avec le numératif un (ex. 21b). Il s'agit là d'un problème qui n’a pas encore été profondément creusé. Il mérite qu'on s'y attarde un peu. On apprendra ainsi un détail précieux qui changera notre conception classique de la distinction massif / comptable et du phénomène de transfert qui lui est subordonné.

\section{Hypothèse du métonymiseur}

\subsection{L'abstractiveur massifiant et le concrétiseur individualisant}

Pour commencer, on considérera un usage assez ancien que la rhétorique place sous le nom de métonymie. Cet usage est surtout très récurrent dans les locutions.

(22) a. Une impératrice doit avoir du cour. (Napoléon I ${ }^{\mathrm{er}}$, Lettres à Joséphine)

b. Ce chien a du nez/ J'ai du nez pour les affaires louches

(23) a. (...) ce sera un magnifique spectacle que la rencontre de deux si fiers courages. (Gautier, ibid.)

b. Son œil avait des éclairs froids à déconcerter l'aplomb des hardiesses. (ibid.)

c. Nous ne disons pas du mal d'elle, nous disons que c'est pas une vertu, ni une intelligence. (Proust, Un Amour de Swan)

d. La pluie venait les interrompre, ou une connaissance qui passait. (Flaubert, Mme Bovary)

e. (...) faire venir M. Canivet de Neufchâtel, qui était une célébrité. (Flaubert, ibid.)

(22) et (23) sont des énoncés figuratifs, car les noms soulignés y figurent en emploi métonymique. Il est clair que ces noms apparaissent sous l'aspect contraire de leurs souscatégories de départ : dans (22), on a affaire à des noms intrinsèquement comptables qui 
sont recatégorisés en massifs et, inversement, dans (23), à des noms intrinsèquement massifs qui sont recatégorisés en comptables. La distribution morphosyntaxique de ces noms avec les marqueurs externes des deux catégories comptable et massive en est la preuve.

A défaut d'un mécanisme de recatégorisation justificatif parmi ceux présentés plus haut, nous pouvons postuler un nouveau mode de recatégorisation qui est d'ordre figuratif - dans la mesure où nous avons affaire à des emplois métonymiques. Nous appellerons ce mode métonymiseur (sur le modèle du trieur, conditionneur, multiplicateur, etc.). Le rôle essentiel du métonymiseur n'est pas d'effectuer la conversion d'une catégorie à l'autre, mais de modifier la structure référentielle du nom sujet au processus de métonymisation. En conséquence, il éclate en deux sous-recatégorisateurs qui, eux, prennent en charge l'explication et la motivation des deux transferts, massif $\rightarrow$ comptable et comptable $\rightarrow$ massif. Nous les appellerons abstractiveur massifiant et concrétiseur individualisant respectivement.

La métonymisation que subissent les noms sous (22-23) affecte en particulier leurs traits sous-catégoriels [+concret] et [+abstrait]. Ce fait se constate dans les deux groupes d'exemples. Ainsi, cœur et nez sont primordialement spécifiés par le trait sous-catégoriel [+concret]. Toutefois, ce trait se trouve modifié sous (22) : cœur et nez ne sont pas sentis comme des noms concrets, mais davantage comme des noms abstraits. Ceci est d'autant plus vrai qu'ils ne dénotent pas des objets discrets, mais plutôt des qualités abstraites, en l'occurrence courage et flair respectivement. Force donc est de conclure que cœur et nez sont recatégorisés en massifs en termes de l'abstractiveur massifiant.

Sur le même modèle, nous invoquerons le concrétiseur individualisant pour rendre compte de la sous-catégorisation comptable des noms donnés sous (23). Un tel mécanisme produit un effet inverse à celui du premier, pour autant qu'il serve à justifier la comptabilisation de noms intrinsèquement massifs. L'effet du concrétiseur individualisant est appuyé par l'interprétation métonymique des noms courage, hardiesse, vertu, intelligence et connaissance. Ceux-ci sont certes des abstraits basiquement massifs. Toujours est-il qu'ils désignent sous (23) des entités concrètes, en l'occurrence les individus auxquels sont attribuées les qualités en question. Leur statut métonymique est donc assuré crucialement par le changement qui affecte leurs traits inhérents : du trait [+abstrait] ils passent au trait [+concret]. Ce fait justifie, en outre, leur distribution avec les marqueurs externes de comptabilité (i.e. deux, des, une, etc.).

L’idée d'éclater le métonymiseur en deux sous-recatégorisateurs répond à un impératif de rigueur et de précision. Compte tenu du changement de la structure référentielle des noms sous (22-23), il est à noter que le caractère comptable et / ou massif n'est pas exclusivement inféodé à la métonymisation. En effet, si celle-ci a pour conséquence d'entrainer généralement le changement des traits sous-catégoriels [+concret] et [+abstrait] intrinsèques aux noms, elle ne peut en aucun cas être tenue responsable des transferts opérés. L'apparition en usage figuratif d'un nom basiquement comptable et / ou massif sous l'aspect inverse n'est pas régie par les traits [+concret] et [+abstrait], pour la simple raison que la distinction massif / comptable caractérise tant les noms abstraits que les noms comptables. C'est, au contraire, le bornage et le non bornage de la région profilée par les noms métonymisés qui déterminent leur sous-catégorisation en comptable et en massif respectivement. Dans ce sens, cœur et nez sont massifs non pas parce qu'ils sont abstraits, mais parce que les qualités qu'ils dénotent par métonymie, à savoir courage et flair respectivement, sont intrinsèquement massives dans l'espace abstrait, qui est ici leur domaine d'instanciation primordial. De même, courage, hardiesse, vertu, intelligence et connaissance acquièrent le trait [+concret] grâce à leur profilation dans l'espace physique, qui est leur domaine d'instanciation primordial sous cet emploi. Toujours est-il que leur caractère comptable ne dépend pas du trait [+concret], mais plus particulièrement des objets intrinsèquement délimités et partant, comptables qu’ils dénotent sous (23). Ceci veut dire, 
en règle générale, que le changement du domaine d’instanciation des noms, comme suite à l'effet du métonymiseur, n'impose aucune contrainte a priori sur la sélection des traits sous-catégoriels des noms.

\subsection{Discussion}

Ce bref exposé appelle deux remarques essentielles, la première concerne le niveau discursif, donc le SN et la seconde, le niveau lexical, donc le N.

(i)- D’une part, il parait légitime, voire fondé et prometteur d'avancer l'hypothèse du métonymiseur. Elle ouvre en effet de nouvelles perspectives de recherche sur le chapitre de la distinction massif / comptable. Elle a l'avantage notamment d'attirer l'attention sur certaines situations discursives où la massification et / ou la comptabilisation de noms basiquement massifs et / comptables s'effectue en termes d'une projection métonymique et / ou métaphorique. Sans pouvoir le confirmer sur pièce pour le moment, il nous semble que la recatégorisation métonymique est à l'origine de certaines tournures massives qui sont en passe de se fixer dans l'usage courant, telles que « avoir de l'œil », " prendre de la carrure», "avoir de la poitrine », "avoir du ventre », etc. La recatégorisation métonymique est surtout très productive dans le langage familier et vulgaire (ex. "avoir de la chatte ", «avoir du cul », etc.). A son tour, la comptabilisation de l'abstrait massif par recatégorisation métonymique n’est pas moins fréquente. On dit communément :

\section{(24) Cet enfant est un espoir / une chance / un malheur / un bonheur, etc., pour nous}

(ii)- D’autre part, si on laisse de côté le SN pour ne tenir compte, dans une perspective strictement lexicale, que les traits lexicaux listés au niveau du N, nous constatons qu'il n’y a plus en fait lieu d'invoquer le processus de métonymisation pour rendre compte de la manifestation des noms sous l'aspect inverse de leurs catégories de départ. En d'autres termes, la conventionnalisation des emplois naguère pragmatiques et, du coup, leur relative stabilité dans la base conceptuelle des noms considérés conduit tout droit au phénomène de la polysémie lexicale. Nous nous emploierons dans la dernière partie à clarifier les tenants et les aboutissants de l'articulation de la distinction massif / comptable à la polysémie lexicale. Comparons pour le moment les énoncés (25a) et (25b) :

(25) a. Je l'ai vu cette nuit, ce malheureux. Sévère, la vengeance à la main. (Corneille, Polyeucte)

b. Il en vint à supposer que c'était peut-être (...) une vengeance de quelqu'un. (Flaubert, Mme Bovary)

L'objectif de cette comparaison est de tenter de tirer au clair la différence subtile qui discrimine les deux emplois, et de parvenir en fin de compte à montrer en quoi les points (i) et (ii) se distinguent l'un de l'autre. Pour aller vite, la différence cruciale entre (25a) et (25b) est celle-là même qui distingue les énoncés figuratifs des énoncés littéraux. On s'accordera ainsi pour dire que (25a) est un énoncé figuratif, parce que le SN la vengeance est en emploi métonymique, et que (25b) est un énoncé littéral, parce que le SN une vengeance n'est pas en usage métonymique (ou métaphorique). Malgré tout, ces SN sont profilés tous deux dans l'espace physique, et sont sentis comme [+concret]. De plus, ils cooccurrent sur la dimension [+comptable], comme en témoigne leur compatibilité avec un et $l a^{4}$. En conséquence, s’il faut conclure à un phénomène de recatégorisation ici, il va falloir invoquer pour chaque cas un mode particulier de transfert. Mais tel ne parait pas être le cas. L'énoncés (25a) ne pose pas de problème à se sujet, et se prête aisément à une explication en termes de la recatégorisation métonymique : le SN la vengeance dénote par métonymie un objet discret (une épée, par exemple), intrinsèquement délimité dans son domaine d'instanciation, l'espace physique. Un tel emploi - si vieux soit l'énoncé exemplifiant - est d'ordre pragmatique ; autrement dit, vengeance au sens de 'épée', ou toute autre arme, est 
une métonymie pragmatique qui se laisse appréhender non sur la base d'un certain savoir lexicographique recouvrant l'ensemble des sens conventionnels relativement stables du lexème vengeance, mais, bien au contraire, sur la base des éléments contextuels de la situation de communication spécifiée. Pour actualiser l'emploi métonymique exemplifié en (25a), nous pouvons construire sur le schème de ce dernier un énoncé comme (26) par exemple :

(26) Prends garde au délinquant que tu as humilié hier; il te cherchait ce matin au restaurant, une vengeance mal dissimulée sous son blouson.

L'interprétation du SN une vengeance sur le mode métonymique comme dénotant le sens de 'une arme' requiert que nous simulions (au sens cognitif du terme) la situation, à l'origine de l'énonciation. Autrement dit, nous devons imaginer la situation en question dans ses moindres détails comme si l'énoncé s’adressait à nous « on-line ». A défaut d'une telle stratégie cognitive, bref, de cette imagerie, nous ne sommes pas très sûrs de comprendre une vengeance au sens de 'une arme'. Il est donc clair que le nom vengeance, un nom abstrait intrinsèquement massif, est versé ici dans la catégorie comptable. Bien entendu, les recatégorisateurs effectuant le transfert massif $\rightarrow$ comptable n'ont aucun droit de cité ici.

Quant à (25b), il est non seulement rétif au trieur et au conditionneur mais à tout recatégorisateur, y compris le concrétiseur individualisant. Pourtant, le SN une vengeance se réalise ici sous l'aspect comptable, et est senti, tout comme le SN une vengeance sous (26), comme [+concret]. Qui plus est, l'espace physique constitue également son domaine d’instanciation primordial. Chose plus curieuse encore, il est également métonymique. Compte tenu d'un pareil "fonds commun », une question de fond se pose : pourquoi ne peut-il pas s’agir dans cet usage tout simplement d'une recatégorisation métonymique ? La réponse tient, en un mot, dans sa conventionnalisation. Mais dans la mesure où tout usage conventionnalisé ajoute au potentiel sémantique des unités lexicales, la polysémie lexicale se trouve catapultée au centre de la problématique de la distinction massif / comptable. La métonymie tient dans ce paysage le devant de la scène, car, comme le souligne Langacker (2013 :70), entre autres, elle alimente régulièrement la polysémie des unités lexicales en vertu des usages métonymiques qui se conventionnalisent et acquièrent, pour ainsi dire, une certaine stabilité.

Il importe peu par ailleurs que la conventionnalisation des usages massifs et / comptables se produisent en termes de métonymisation, de métaphorisation ou de tout autre mécanisme. Plus essentiel est plutôt ce fait que les usages en question correspondent (dénotent) à des sens relativement assez stables pour être identifiés comme des acceptions d'un même vocable. On l'aura remarqué, la distinction massif / comptable, contrairement à ce que nous apprend la littérature d'inspiration aristotélicienne sur le sujet, ne forme pas une opposition binaire divisant, pour ainsi dire, le lexique en deux catégories exclusivement opposées, celle des massifs et celle des comptables ${ }^{5}$. Bien au contraire, la complexité conceptuelle des matrices des noms nous amène à considérer le massif et le comptable comme deux catégories conceptuelles qui ne s'opposent qu'en elles-mêmes. Ceci revient à dire qu'elles ne sont pas nécessairement exclusives dans les expressions linguistiques qui les instancient. Compte tenu de ce fait, bien de cas, traditionnellement identifiés et traités en tant que faits de recatégorisation, se révèlent être en réalité des faits de polysémie lexicale. L'objectif de la section suivante est de tenter d'apporter un éclairage sur ce rapport, non encore suffisamment creusé, qui articule la distinction massif / comptable à la polysémie lexicale.

\section{La distinction massif / comptable sur le mode polysémique}


Il doit être clair pour le moment que la recatégorisation, fût-elle d'ordre figuratif, constitue un phénomène pragmatique et non lexical. Elle répond en principe au besoin de conceptualiser « on-line » une réalité massive et / comptable donnée dans le giron inverse. Ceci détermine dans une large mesure sa grande productivité. Aussi peut-on, par forçage (un terme présentement en vogue dans la littérature) 'couler' n’importe quel objet comptable dans le moule du massif (voiture $\rightarrow$ de la voiture ; fauteuil $\rightarrow$ du fauteuil, etc.). Les expressions massives de la voiture et $d u$ fauteuil, ne semblent naturellement dénoter aucun référent conventionnel. En conséquence, leur interprétation est hautement marquée, et est construite en fonction du contexte d'énonciation spécifié. En revanche, l'interprétation d'autres expressions du même type n'est pas entièrement assujettie aux contextes d'énonciation. Ainsi, les expressions du vélo, du cheval, de la moto, du violon, etc., sont comprises en dehors d'une situation d'énonciation. Qui plus est, elles ne sont pas senties comme transférées du comptable au massif, dans la mesure où les sens associés aux objets comptables de départ (un vélo, un cheval, une moto, un violon) ne sont pas identiques aux sens des expressions massives en question. Plus curieux encore est le fait que leur sens massif est compris en dehors de leur distribution avec le partitif : vélo, cheval, moto, violon ont, entre autres, le sens comptable de 'objet discret' aussi bien que le sens massif de 'pratique, activité'. On en conclut alors que ces noms sont des vocables associés chacun à deux acceptions (à titre limitatif, bien entendu). Ces acceptions dénotent deux référents qui sont opposés sur la dimension [+comptable] et [+massif]. On l'aura constaté, la « coexistence paradoxale " ${ }^{6}$ des deux traits dans la même base conceptuelle des noms ne semble s'expliquer qu'en termes d'une polysémie lexicale. Il s'ensuit que les traits massif et comptable sont 'co-listés' au niveau lexical du nom et constituent, de ce fait, des propriétés lexicales.

L’intérêt de mettre la polysémie lexicale au cœur de la distinction massif / comptable est de tenter de lever une confusion qui règne toujours dans le domaine de la recatégorisation (cf. G. Numberg et A. Zaenen, 1997). Une telle tentative se doit de séparer les emplois massifs et / ou comptables conventionnalisés des emplois construits en fonction des contextes d'énonciations ${ }^{7}$. L'attitude adoptée ici repose sur un présupposé théorique bien défini : tout en accordant crédit à la thèse constructiviste selon laquelle le sens est une construction dynamique, négociable en contexte, elle reconnait comme allant de soi que le sens des unités lexicales est défini, conventionnel et relativement assez stable pour être identifié comme tel, et permettre ainsi aux locuteurs de le négocier et le renégocier en situation de communication. Il est vrai qu'à défaut d'une telle position, il est tout juste inutile de parler de polysémie lexicale.

Nous noterons par ailleurs que la polysémie lexicale affecte tant les comptables que les massifs. En effet, de même que l'extension de sens des comptables basiques peut déboucher sur une / des acception(s) massive(s), de même l'extension de sens des massifs basiques peut aboutir à une / des acception(s) comptable(s). Compte tenu de cette caractéristique, une mise au point s'impose : notre analyse repose sur les deux postulats suivants :

- le nom, source de l'extension massive, est basiquement comptable

- le nom, source de l'extension comptable, est basiquement massif

De plus, elle ne prend pas en compte seulement le niveau du N, mais également le niveau du SN. Du coup, elle s’appuie, pour déterminer le caractère polysémique de l'unité lexicale considérée, sur l'apport syntaxico-sémantique des marqueurs externes de la distinction massif / comptable.

\subsection{Polysémie lexicale comptable $\rightarrow$ massif. Les noms d'instruments de musique}

Pour tester l'hypothèse de la polysémie lexicale comptable $\rightarrow$ massif, nous nous limiterons à l'examen des noms d'instruments de musique ${ }^{8}$. Ces noms ont pour dénominateur 
commun d'impliquer, à la suite d'un transfert de type métonymique (i.e. le métonymiseur), des acceptions massives. Le phénomène est, en effet, si régulier qu'il semble se prêter mieux à une description en termes d'une polysémie lexicale systématique.

Les noms d'instruments de musique présentent une caractéristique commune essentielle sur le chapitre de la relation comptable-massif : ils s'emploient en tant que [+comptable] dans le sens de 'objet discret' et en tant que [+massif] dans le sens de 'musique ou activité / pratique de musique' produite ou accomplie sur la base de l'objet luimême. Ces deux emplois sont très fréquents dans l'usage courant. Aussi peut-on dire (27a) ou (27b) selon qu'on veut référer à 'instrument' [+comptable] ou à 'activité' [+massif] :

(27) a. J'ai acheté un violon / un piano / une guitare /une trompette, etc. b. Je joue du violon / du piano / de la guitare / de la trompette, etc.

Dans le cadre stricte d'une distinction tranchée massif / comptable, ces emplois impliquent le postulat suivant: dans la mesure où 'instrument' [+comptable] est la source de dérivation de 'activité' [+massif], la massivité des SN sous (27b) ne peut s'expliquer qu'en termes d'une recatégorisation. Ce postulat ne tient pas la route : la comparaison de (28) avec (29) fait ressortir une différence sémantico-référentielle cruciale :

(28) Cet amateur du rock joue de la guitare tout le temps

(29) Dans ce magasin, il y a de la guitare partout

Dans les deux énoncés, on a la même expression massive de la guitare. Mais cette expression n'a pas le même sens et, du coup, ne dénote pas le même référent. Bref, il s’agit de deux expressions bien distinctes - l'identité du signifiant est une affaire d'homonymie. Conclure à des faits de recatégorisation sous (28) et (29) exige que soient satisfaites deux conditions, que nous promouvons ici au rang de tests.

(1)- Test du recatégorisateur justificatif : en cas de recatégorisation, on doit identifier le mécanisme à la base du transfert effectué.

(2)- Test de la concordance des traits lexical et discursif: dans le cas d'une recatégorisation, on relève une concordance ente le trait du $\mathrm{N}$ et le trait du SN.

La dernière condition rappelle la position de Weinrich (1966), Kleiber (1990, 1994 et 1999), entre autres, selon laquelle le nom massifié préserve le trait [+comptable] au niveau du SN, si bien qu'il n'y a pas discordance entre son trait lexical et son trait discursif.

L’énoncé (29) remplit les deux conditions. Premièrement, l'expression de la guitare répond parfaitement à une massification en termes du multiplicateur : cette recatégorisation est d'ordre pragmatique, car l'interprétation du SN de la guitare dans le sens d'une pluralité d'occurrences de l'objet guitare est tributaire du contexte global de l'énonciation. Elle est d'autant plus pragmatique qu'elle est susceptible de changer en fonction de la variation des contextes. Ainsi, de la guitare sous (29) peut également référer à une réalité broyée, impliquant en conséquence l'opération du broyeur. Cette interprétation peut émerger, à titre d'exemple, à la vue de guitares broyées jonchant le sol d'un magasin vandalisé. Deuxièmement, la condition de la concordance est également remplie. En effet, le trait [+comptable], intrinsèque à la conception basique de l'objet guitare, est préservé sous l'emploi massive de l'expression de la guitare : le trait [+comptable] demeure inhérent au SN massifié, parce que - s'agit-il de multiplication ou de broyage - l'expression de la guitare désigne toujours des objets comptables.

Inversement, l'expression de la guitare sous (28) ne satisfait à aucune des deux conditions précitées. En premier lieu, le référent dénoté par de la guitare n’est ni multiplié ni broyé. On est tenté par contre d'invoquer le métonymiseur, et plus particulièrement, le concrétiseur massifiant pour rendre compte de l'apparition du nom guitare sur la dimension [+massif]. A première vue, rien ne s’y oppose, puisque cet usage résulte effectivement de la métonymisation de l'objet guitare : l'expression de la guitare dénote un référent non borné dans l'espace abstrait, à savoir 'activité de musique'. Mais, à y regarder de près, la 
compréhension de de la guitare au sens de 'activité de musique’ n'est pas du tout marquée, et partant, ne ressortit pas à des contextes d'énonciations particuliers. Donc, elle ne coïncide pas avec une recatégorisation pragmatique. L'énoncé (28) - de même que l'énoncé (27b) - n'est ni ambigu ni moins usuel, et se laisse, par-dessus tout, comprendre en dehors d'une situation physique de communication. Force est de constater que le sens 'activité de musique', associé par métonymie au nom guitare, est conventionnalisé sur la dimension [+massif]. Le trait massif est donc une propriété lexicale du nom guitare. La preuve en est que celui-ci peut se manifester sur la dimension [+massif] en dehors de la structure partitive, comme c'est l'usage avec des expressions du type de "aimer la guitare ", «apprendre la guitare », etc. En second lieu, la condition de la concordance n'est pas remplie non plus. C'est que le trait discursif [+massif] manifesté au niveau du SN (de la guitare) est en discordance avec le trait lexical [+massif] du nom (guitare). En d'autres termes, le trait discursif [+massif] correspond à l'acception massive 'activité de musique' et non à l'acception comptable 'instrument'.

Le cas de guitare n'est pas un cas isolé ; il est plutôt représentatif du paradigme tout entier. Aussi les noms d'instruments de musique manifestent-ils sur le mode polysémique comptable $\rightarrow$ massif une régularité qui est telle que leur comportement est prédictible. Cette prédictibilité nous conduit à postuler à leur base une polysémie lexicale systématique, pour autant que tout nom désignant un instrument de musique possède systématiquement un autre sens par référence à l'activité / pratique de la musique impliquée, comme on le voit dans (30) :

(30) - Flûte 'instrument' (une flûte [+comptable]) $\rightarrow$ ‘activité / musique de la flûte' (de la flûte [+massif])

- Violon 'instrument' (un violon [+comptable]) $\rightarrow$ 'activité / musique du violon' (du violon [+massif]

\subsection{Polysémie lexicale massif $\rightarrow$ comptable}

La polysémie lexicale massif $\rightarrow$ comptable est également envisageable pour les noms intrinsèquement massifs qui bénéficient d'acceptions sur la dimension [+comptable]. Nous tenterons d'examiner dans ce qui suit le comportement identique de deux types de noms qui semblent entériner le bien-fondé de ce postulat, en l'occurrence les noms concrets de matières et un paradigme particulier de noms abstraits, les noms abstraits intensifs.

\subsubsection{La polysémie lexicale massive $\rightarrow$ comptable des noms de matières}

Ce paradigme comporte les noms qui réfèrent à la fois à des matières intrinsèquement massives et aux objets intrinsèquement comptables qui sont fabriqués sur la base de ces matières mêmes. Notre propos porte exclusivement sur les matières qui entrent dans la fabrication d’objets discrets. Considérons à titre d'illustration les énoncés suivants :

(31) a. Pendant la guerre on a fabriqué du caoutchouc synthétique

b. Chez le fleuriste, mon père a acheté un caoutchouc pour la fête de ma mère (Obadia, 1976 : 77)

c. Elle me voyait chaussant mes caoutchoucs américains. (Proust, cité par le P. Robert)

(32) a. C'est en Lorraine qu'on extrait la plus grande partie du minerai de fer français. (Obadia, ibid.)

b. Dans cette quincaillerie, tu trouveras des fers de toutes sortes : à repasser, à cheval, à friser, à gaufrer ... (Obadia, ibid.) 
On assiste sous ces exemples à deux emplois différents des noms caoutchouc et fer : en (31a) et (32a), caoutchouc et fer dénotent les substances caoutchouc et fer respectivement ; bref, ils apparaissent sur la dimension [+massive]. En (31b-c) et (32b), en revanche, ils réfèrent à des objets discrets fabriqués à partir des substances dénotées ; donc, ils se manifestent sur la dimension [+comptable]. Tout le problème est de déterminer si cette différence d'emploi est une question de polysémie lexicale ou, au contraire, de recatégorisation.

Notre position est claire sur ce point : les emplois comptables des noms de matières correspondent à des usages métonymiques qui se sont conventionnalisés sur la dimension [+comptable]. Ces noms sont donc polysémiques en ce qu'ils regroupent généralement deux acceptions distinctes sur les dimensions [+massif] et [+comptable]. Pour corroborer cette hypothèse, il nous incombe de montrer pourquoi les emplois comptables des noms de matières ne correspondent pas à une recatégorisation. Pour ce faire, nous invoquerons les deux conditions (1) et (2) présentées plus haut. Comparons, pour illustrer ce point, (31a-c) et (32b) à (33a-b), cités par T. Vermote (2014) :

\section{(33) a. L'approvisionnement s'effectue uniquement avec des laines françaises (...) \\ b. (...), c'est la certitude d'un tissu de qualité à un prix imbattable}

Certes, les noms laine et tissu sont basiquement massifs. Mais sous (33), ils se manifestent sous l'aspect comptable. On reconnait d'un accord commun que les SN des laines et un tissu réfèrent à des variétés de laine et de tissu respectivement. En conséquence, ils se présentent ici comme recatégorisés en comptables. La preuve en est que leur transfert ne se traduit ni par une variation totale de sens ni par une certaine discordance entre le trait du N et celui du SN. En somme, le trait lexical [+massif] des substances laine et tissu est préservé sous leur transfert discursif en SN comptables des laines et un tissu. Tel étant le cas, la seconde condition, un corollaire de la première, est automatiquement remplie : le mécanisme qui justifie le passage massif $\rightarrow$ comptable est le trieur universel. Sur le plan sémantique, le choix du trieur, au lieu d'un autre mécanisme, est principalement motivé par le sème discursif (i.e. le virtuème) [+type] qui s’ajoute aux sémantèmes des noms concernés.

A la différence de (33), les emplois comptables sous (31b-c) et (32b) ne vérifient pas la condition de concordance entre le trait du $\mathrm{N}$ et le trait du SN. A y regarder de plus près en effet, le trait discursif [+comptable] de un caoutchouc, mes caoutchoucs, des fers est en totale discordance avec le trait lexical [+massif] des noms correspondants : il est difficile, sinon impossible, d'identifier une quelconque massivité sous les emplois comptables. Du coup, la manifestation du trait discursif [+comptable] dans les SN un caoutchouc, mes caoutchoucs, des fers est motivée à la base par une polysémie lexicale. Ceci revient à dire que leur trait [+comptable] est une propriété lexicale. Ensuite, en l'absence d'une concordance entre les traits du $\mathrm{N}$ et du SN, la seconde condition, celle du recatégorisateur justificatif du transfert effectué, n'est pas satisfaite aussi. Le conditionneur est naturellement écarté pour l'aspect d’homogénéité qu’il introduit au niveau du référent dénoté (cf. T. Vermote). Reste à tester le collecteur et le métonymiseur. Le collecteur est postulé par T. Vermote pour élucider plus particulièrement la manifestation comptable des noms de matériaux sous les emplois qui nous concernent ici. Il semblerait ainsi, d'après l'idée du collecteur, que la collection des objets dénotés par les SN vos cuirs, les cotons, ses métaux, exemplifiés dans (34) répond à un transfert massif $\rightarrow$ comptable :

(34) a. Entretien du cuir : pour détacher (...), et imperméabiliser vos cuirs

b. Utiliser un détachant qui préserve les couleurs sur les cotons colorés

c. A combien peut-on revendre ses métaux chez les ferrailleurs?

L'idée du collecteur prête le flanc à deux critiques essentielles. D'une part le collecteur échoue d'emblée sur les écueils des exemples (31b-c) où les structures référentielles des SN 
un caoutchouc et mes caoutchoucs américains ne coïncident pas avec l'idée de collection au sens de 'ensemble d'objets peu spécifiques'. Ces noms ont pourtant à la base les mêmes données que "vos cuirs », "les cotons colorés » et "ses métaux» : ce sont des noms de matériaux intrinsèquement massifs, et ils se manifestent discursivement sur la dimension [+comptable]. D'autre part, le collecteur renferme une certaine contradiction : il dit par exemple que les SN pluriels vos cuirs, les cotons colorés et ses métaux n'ont pas de correspondants singuliers, c'est-à-dire qu'ils ne correspondent pas aux singuliers un cuir, un coton coloré et un métal respectivement. Ceci a pour avantage certes d'écarter l'éventualité d'un transfert en termes d'un triage qualitatif. Mais dans le même temps, l'auteur l'envisage comme un transfert d'un type sémantique. Il nous semble, sur ce dernier point, que le collecteur parait faire raison de l'idiosyncrasie d'un fait spécifique de la polysémie lexicale massif $\rightarrow$ comptable.

Quant au métonymiseur, il est certes à l'origine des emplois comptables des noms de matières donnés tant sous (31b-c) et (32b) que sous (34a-c). Mais il l'est seulement du point de vue diachronique. En effet, dans la mesure où ces usages se sont conventionnalisés dans l'usage courant sur la dimension [+comptable], ils ne sont pas appréhendés synchroniquement comme des métonymies pragmatiques - dont l'assimilation nécessite un contexte particulier de communication. Du coup, leur trait [+comptable] constitue une propriété lexicale, listée au niveau du N.

Un fait est curieux par ailleurs : les noms de matières, abstraction faite de certains ${ }^{9}$, manifestent une régularité systématique sur le mode de la polysémie massif $\rightarrow$ comptable. Nous en sommes amené à postuler à leur sujet une polysémie lexicale systématique de type métonymique et / ou métaphorique indiquant que s'il y a le sens [+massif] 'matière', il y a également possibilité d'avoir le sens [+comptable] 'objet travaillé dans cette matière'. Tel nous semble être le cas des noms de matières du type de zinc, fer, cuivre, argent, etc., comme l'illustrent les exemples (35-38) :

(35) Les faux marbres et les zincs dorés du vestibule (Zola, Pot-Bouille)

(36) L'homme est né libre, et partout il est dans les fers (Rousseau, Du contrat social)

(37) Les cuivres rutilaient : casseroles de toutes tailles, chaudrons, écumoires, bassines, bassinoires (Beauvoir, Mémoires d'une jeune fille rangée)

(38) (...) une éraillure sur les argents et les cuivres... (Mirbeau, Le Journal d'une femme de chambre)

Les SN les zincs, les fers, les cuivres et les argents peuvent en théorie désigner par métonymie et / ou métaphore tout objet fabriqué des matières correspondantes. Toujours est-il que dans les exemples (35-38), ces SN dénotent des sens bien définis : zincs sous (35) désigne des objets en zinc, plus précisément, 'des feuille de zinc', fers sous (36), un objet en fer, en l'occurrence 'une chaine, des menottes', cuivres sous (37), des objets en cuivre, en particulier ' les ustensiles ou objets d'ornement en cuivre' et enfin argents sous (38), des objets en argent, à savoir 'la vaisselle en argent'. Ces sens sont synchroniquement stables dans l'usage courant, si bien qu'il n'y a pas lieu de conclure à leur propos à un certain transfert, de quelque type qu'il soit. La polysémie lexicale massif $\rightarrow$ comptable demeure dans ce sens une alternative très prometteuse à même d'apporter de nouveaux éclairages sur la nature de la corrélation des traits massif et comptable, ainsi que sur la complexité souscatégorielle des noms en général.

\subsubsection{La polysémie lexicale massif $\rightarrow$ comptable des noms abstraits intensifs}

La problématique des noms abstraits sur le mode de la distinction massif / comptable n’a pas passé inaperçu (Lemghari, 2010a). Sans prétendre à une certaine exhaustivité, nous voudrions attirer l'attention ici sur la complexité de la base conceptuelle d'un paradigme particulier de noms abstraits, communément appelés, depuis Van de Velde (1995), noms 
intensifs. Le choix de « intensif » pour qualifier cette classe est motivé par une conception des abstraits qui est identique à la conception des substances concrètes massives. Une telle identité se traduit linguistiquement par une distribution morphosyntaxique similaire. Le terme intensif nous semble employé par l'auteur dans son acception philosophique originelle de grandeur intensive, un concept qui décrit la qualité de ce qui ne se laisse pas mesurer et qui, du coup, admet une évaluation en termes de degrés variables. Les abstraits intensifs sont donc conceptuellement des massifs fondamentaux. Ce sont les noms de qualité, les noms d'état et les noms de sentiment. Le problème que soulève à nos yeux les noms abstraits en général ne se ramène pas seulement à la question de savoir s’ils ont ou non des emplois comptables, mais surtout à celle de déterminer la nature de ces emplois. En d'autres termes, il convient de montrer si la manifestation des noms abstraits massifs, tout comme les noms concrets massifs, sur la dimension [+comptable] est le corollaire d'une recatégorisation ou le fait d'une polysémie lexicale.

Pour aller vite, la revue de la littérature sur le sujet montre que l'emploi des noms abstraits massifs en comptables correspond à un transfert de type qualitatif. Pourtant, pris sous la parité du comportement morphosyntaxique, les noms abstraits massifs intensifs révèlent une certaine régularité sur la dimension [+comptable]. Beauseroy (2008) promeut cette régularité au rang d'une généralisation de type morphosyntaxique fondée sur le parallélisme qu'elle établit entre «l'opposition non pluralisable / pluralisable et l'opposition lecture de nom statif / lecture d'occurrence ». En un mot, les noms abstraits intensifs manifestent régulièrement deux comportements morphosyntaxiques distincts corrélés à deux lectures distinctes, une lecture stative (massive) et une lecture d'occurrence (comptable). Bref, ils sont en emploi massif ou en emploi comptable selon qu'ils acceptent ou non la pluralisation. Une question se pose alors: vu leur régularité sur le plan morphosyntaxique, les deux lectures distinguées correspondent-elles, sur le plan lexical, à une polysémie systématique ? Tout porte à le croire en effet, car, eu égard aux lectures présupposées, rien n’empêche de postuler à la base du paradigme une polysémie lexicale systématique du type de: 'si le nom abstrait intensif a un sens ' $x$ ' sur la dimension [+massif], il a également le sens 'occurrence de $x$ ' sur la dimension [+comptable]'. Mais, convient-il de rappeler, sur le plan sémantique, le paradigme des noms abstraits intensifs n'est pas aussi homogène que le suggère l'analyse de Beauseroy. Nous présenterons cidessous un bref exposé de leurs caractéristiques distinctives sur le mode massif $\rightarrow$ comptable.

Nous rappellerons, avant d'aller plus loin, que la base conceptuelle des noms est composée d'un faisceau de domaines cognitifs. Ces domaines forment, pour ainsi dire, le contexte informatif des noms. Une des implications corrélatives de cette conception est d'admettre que les noms présentent une complexité sous-catégorielle au niveau conceptuel, laquelle complexité est encodée dans la structure linguistique. Ainsi, pour reprendre l'exemplification de Langacker (1991), les noms bip et flash impliquent une combinaison de deux domaines cognitifs. Bip évoque à la fois le temps et la fréquence, autrement dit, deux domaines où il se prête comme borné en raison, d'une part, de sa durée courte et, d'autre part, de sa modélisation comme tonalité. Flash, tout comme bip, se définit aussi bien dans le domaine temporel que dans le champ visuel, à une différence près : il est normalement borné dans le premier, mais pas nécessairement dans le second. La conclusion que nous en tirons est qu'un nom massif et / ou comptable ne préserve pas forcément son trait [+massif] et / ou [+comptable] dans tous ses domaines d'instanciation. Il nous semble que nous gagnerons davantage à justifier le postulat de la polysémie lexicale massif $\rightarrow$ comptable pour les noms abstraits intensifs sur la base de la pluralité des domaines cognitifs caractéristiques de leurs bases conceptuelles. Cette façon de faire nous conduira à dévoiler le rôle latent que remplit la variation des domaines cognitifs dans l'emploi et l’interprétation des noms. 


\subsubsection{Bases conceptuelles des noms abstraits intensifs}

Dans un souci d’homogénéisation, Beauseroy (ibid.) place sous le nom «statif » les trois types de noms abstraits intensifs distingués. Suivant en cela Van de Velde (1995), Flaux et Van de Velde (2000), elle appuie leur caractère statif sur la base de l'identité de leur comportement morphosyntaxique : les noms abstraits intensifs ne s'emploient pas avec l'adverbe beaucoup sous une lecture durative ou fréquentative, de la même manière que les verbes et / ou les adjectifs auxquels ils sont apparentés. Les exemples de Beauseroy (ibid. : 2390), que nous reprenons sous (39-41), illustrent ce point :

(39) Noms de sentiment

a. Les hommes donnent beaucoup d'amour à leurs animaux domestiques

b. = Les hommes aiment intensément leurs animaux domestiques

c. $\neq$ Les hommes aiment pendant longtemps / souvent leurs animaux domestiques

(40) Nom de qualité

a. Nos hôtes nous ont reçus avec beaucoup de gentillesse

b. = Nos hôtes étaient profondément gentils

c. $\neq$ Nos hôtes étaient gentils pendant longtemps / souvent

(41) Nom d'état

a. Marie a ressenti beaucoup de fatigue après le marathon

b. = Marie était extrêmement fatiguée

c. $\neq$ Marie était fatiguée pendant longtemps / souvent

Comparés aux noms extensifs danse, jardinage et voyage, exemplifiés sous (42), le contraste de comportement des intensifs et des extensifs est si évident que notre conviction est immédiatement conquise :

(42) Noms abstraits extensifs

a. Marie a fait beaucoup de danse $\quad \approx$ Marie a dansé longtemps / souvent

b. Pierre a fait beaucoup de jardinage $\approx$ Pierre a jardiné longtemps / souvent

c. Max fait beaucoup de voyages $\quad \approx$ Max voyage souvent

Tout compte fait, la différence entre les abstraits intensifs et les abstraits extensifs se ramène à la propriété d'exclure ou d'admettre une extension temporelle, durative ou fréquentative. Cette conclusion nous parait logique dès lors quand on aborde les noms abstraits, toutes classes confondues, du point de vue de leur typologie générale. Mais l'examen de leur comportement sémantico-syntaxique à la lumière du postulat de la complexité cognitive de leurs bases conceptuelles nous pousse à revoir leur conception classique. Ainsi, la régularité de la manifestation des noms abstraits intensifs sur la dimension [+comptable] en lecture d'occurrence soulève une question légitime : les noms abstraits intensifs ne sont-ils pas également des noms extensifs ? Nous tenterons d'apporter un élément de réponse à cette question en examinant les bases conceptuelles des trois sousclasses.

Les trois types de noms s'instancient primordialement dans des espaces cognitifs distincts : l'espace qualitatif est le domaine d'instanciation des noms de qualité, l'espace émotif, celui des noms de sentiment et l'espace affectif ${ }^{10}$, celui des noms d'état. Nous rappellerons que la manifestation massive et / ou comptable de ces noms ne dépend pas nécessairement de la nature de l'espace d’instanciation impliqué, mais plus précisément du bornage et / ou non bornage de l'entité profilée. Ainsi, en dépit de la différence de leurs domaines cognitifs basiques, les trois types de noms profilent, chacun dans son domaine respectif, des entités intrinsèquement non bornées ; d'où leur sous-catégorisation [+massif], comme il ressort de (43-45) :

(43) Il a montré de la patience pour elle (nom de qualité) 
(44) Il a montré de l'admiration pour elle (nom de sentiment)

(45) Il a montré de la colère pour elle (nom d'état)

Malgré leur primordialité, les espaces basiques ne forment pas à eux seuls la totalité de la structure informative des noms. Celle-ci peut en effet consister en la combinaison de plusieurs domaines cognitifs. Toujours est-il que ces domaines n'acquièrent pas le même degré de centralité et de conventionnalisation: certains domaines pour certains noms généralement pour des rasions socioculturelles - sont plus conventionnels et plus centraux que le reste des domaines de leurs bases conceptuelles. Sous cet angle, les bases conceptuelles des noms de qualité / sentiment / état sont co-structurées, en plus de leurs domaines cognitifs basiques respectifs, par deux autres domaines, en l'occurrence le domaine physique et le domaine temporel, qui, parfois, fusionnent en un seul domaine spatio-temporel. En règle générale, le comportement morphosyntaxique des noms de qualité / sentiment / état est prédictible sur la base de cette propriété commune qu'ils ont d'être profilés communément dans les domaines physique et temporel. En d'autres termes, leur grandeur qualitative / émotive / affective est réifiée en grandeur physique ou grandeur temporelle ou encore grandeur spatio-temporelle selon qu'ils sont instanciés dans le domaine physique ou le domaine temporel ou encore le domaine spatio-temporel.

Nous commençons par les noms de qualité pour souligner une différence qui les sépare des autres noms. Ils sont, nous semble-t-il, les seuls noms intensifs qui s'instancient régulièrement dans le domaine spatio-temporel sous leur interprétation en acte. Cette propriété est due à la conception de l'entité 'acte' elle-même : un acte, de quelque type qu'il soit, s'accomplit dans le temps et l'espace. Considérons à titre d'illustration les exemples suivants :

(43) a. Ce film nous inspire des folies

b. Paulette n'a pas oublié les imprudences de son mari

c. Le président a échangé des politesses avec son invité d'honneur

En général, les actes qualifiés, malgré leur variabilité sur la dimension [ \pm concret], sont perçus comme instanciés primordialement dans le domaine spatio-temporel. Ainsi, les SN des folies, les imprudences et des politesses, en tant qu'idées [ \pm abstrait] (folies), comportements [-concret] (imprudences), paroles [ \pm concret] (politesses), ou toute autre chose, s'accomplissent dans l'espace physique dans le même temps qu'elles délimitent un / des intervalle(s) dans l'espace temporel. Leur conception de noms comptables découle de nos connaissances expérientielles qu'un acte est intrinsèquement borné dans les deux dimensions, physique et temporelle.

A part cette différence, les noms de qualité / sentiment / état se profilent régulièrement dans les domaines physique et temporel. Nous remarquons tout de même qu'ils ne tendent pas à privilégier les mêmes domaines d'instanciation. Tout bien considéré, leur base cognitive commune consiste dans leur profilation régulière dans l'espace temporel. Soit les exemples suivants :

(46) On ne te pardonnera pas les injustices commises sous vos ordres (nom de qualité)

(47) Elle a fait des dépressions ces deux dernières années (nom d'état)

(48) Je lui ai expliqué la cause de mes haines (nom de sentiment)

Le statut comptable des occurrences les injustices, des dépressions et mes haines ressortit en principe à leur conceptualisation en tant que procès épisodiques s'accomplissant dans l'espace temporel. Chaque occurrence de ces noms occupe une fraction de temps qui comporte en elle-même le principe de sa limite. La pluralisation est corrélative ici de la répétition de l'occurrence un certain nombre de fois. 
Reste l'espace physique. Les noms de qualité / sentiment / état s'y manifestent sur la dimension [+comptable]. Leur lecture d’occurrence est assurée en général par les entités intrinsèquement comptables auxquelles ils réfèrent dans ce domaine. Considérons :

(49) Noms de qualité

a. Les armées anciennes n'enrôlaient que les courages et les hardiesses

b. Les talents du cinéma moderne sont très chers

c. Les génies de notre époque sont la cause de notre malheur

d. ?? J'ai rencontré deux justices dans ce tribunal / deux imprudences sur mon chemin

(50) Noms de sentiment

a. Paul a connu trois amours dans sa vie

b. Sa beauté excite les haines et les jalousies de son entourage

c. ?? Il a été applaudi (hué) par les estimes (les dégoûts) présents au spectacle

(51) Noms d'état
a. Il n'aime pas échanger des propos avec les connaissances du voisinage
b. Ce plaisantin fait des courbettes aux beautés qui passent devant sa boutique
c. La conférence a réuni les célébrités du pays
d. ?? Jai connu des angoisses (= personnes angoissées), des fatigues (= personnes fatiguées), des concentrations (= personnes concentrées), etc.

Eu égard aux exemples douteux (49d), (50c) et (51d), nous constatons que les noms de qualité / sentiment / état ne s'instancient pas régulièrement dans l'espace physique. En effet, en dépit de sa centralité dans leur matrice cognitive, il n’est pas évoqué avec la même régularité que l'espace temporel. Nous remarquons de plus que le mécanisme, à l'origine de ces emplois, est le même, en l'occurrence, le métonymiseur. Abstraction faite de leurs sous-classes respectives, ces noms dénotent par métonymie des objets discrets, visiblement [+concret] : courages, hardiesses, haines, jalousies, connaissances, beautés et célébrités renvoient aux individus, objets des qualités / sentiments / états concernés. Ils acquièrent, du coup, sur la dimension [+comptable] des acceptions différentes de celles qu'ils ont sur la dimension [+massif].

Ce bref examen du comportement massif / comptable des trois sous-classes à la lumière de la différence de leurs domaines d'instanciation aboutit à deux conclusions. D’une part, pris individuellement, les noms de qualité / sentiment / état ne vérifient que l’hypothèse de la polysémie lexicale massif $\rightarrow$ comptable, dans la mesure où il existe au sein de chaque sous-classe des noms qui sont rétifs à l'interprétation en occurrences comptables dans chacun des domaines cognitifs distingués : par exemples, les (49d), (50c) et (51d) excluent l'espace physique. D’autre part, pris globalement, ces noms vérifient l'hypothèse de la polysémie lexicale systématique massif $\rightarrow$ comptable en ce qu'ils admettent dans l'ensemble des domaines cognitifs distingués des emplois massifs et des emplois comptables. Compte tenu de cette précision, on peut formuler de la manière suivante l'hypothèse de la polysémie systématique pour les noms de qualité / sentiment / état : 'si le nom abstrait intensif a un sens ' $x$ ' sur la dimension [+massif], il a également dans l'un de ses domaines d'instanciation le sens 'occurrence de x' sur la dimension [+comptable]'.

\subsubsection{Corrélation des domaines cognitifs et polysémie lexicale massif $\rightarrow$ comptable}

La prise en ligne de compte de la notion de domaine cognitif a l'avantage de dissiper le flou qui règne autour de certains emplois. Par exemple, Beauseroy (ibid. : 2396) fait remarquer que la lecture d'occurrence n'est pas disponible pour le nom de qualité courage, comme en 
témoigne l'agrammaticalité de (52). Nous soulignerons tout de même que (53) est bel et bien acceptable :

(52) * Paul a montré des courages quand il le fallait

(53) Le communisme (...) un " idéal » supérieur à celui de la patrie, enrôlant les courages et les dévouements des divers pays pour une cause commune (Gide, Journal)

Nous en déduisons que l'inacceptabilité de (52) n'est pas la preuve de l'absence de lecture d'occurrence pour le nom courage ; l'acceptabilité de (53) en est le témoignage. En somme, la lecture d'occurrence pour le nom courage - de même que pour le nom dévouement - est chose possible, et implique que le domaine d'instanciation est l'espace physique et non l'espace temporel (les courages et les dévouements réfèrent ici à des entités concrètes, en l'occurrence les personnes courageuses et les personnes dévouées). Il s'ensuit que la polysémie massif $\rightarrow$ comptable pour le nom courage met en corrélation le domaine qualitatif (emploi massif) et le domaine physique (emploi comptable).

Notons que la lecture d'occurrence pour les noms de qualité / sentiment / état n'est pas restreinte à l'instanciation de ces noms dans un domaine cognitif exclusif. Certains noms peuvent évoquer sous leurs emplois comptables aussi bien l'espace physique que l'espace temporel, donnant ainsi lieu à deux occurrences comptables associées à autant d'acceptions. Tel est le cas, à titre d'exemple, du nom de sentiment amour, comme l'illustrent (54) et (55) :

(54) Il a trois enfants, donc trois amours

(55) On a beaucoup parlé des amours du président avec sa secrétaire

Dans (54), le nom amour est [+comptable]. Il est instancié dans l'espace physique. Il est [+concret] ; il réfère à des objets discrets. Dans (55), il est également [+comptable]. Mais à la différence de (54), il est profilé dans l'espace temporel. De ce fait même, il est [-concret]. La nuance, sinon la différence de sens entre les deux occurrences du nom amour est claire : sous (54), le nom amour signifie 'objets de l'amour'. Sous (55), il signifie 'épisodes ou série des expériences d'amour'.

Par ailleurs, dès lors que nous admettons que les noms de qualité / sentiment / état ont des extensions temporelles, nous soulevons le problème suivant: si ces noms ont des extensions temporelles sous leurs emplois d'occurrences, ils doivent vérifier le test de la lecture durative et / ou fréquentative. En principe, si nous acceptons le postulat que les abstraits intensifs sont co-structurés au niveau lexical par les traits massif et comptable, nous devons aussi admettre qu'il n'y a aucune raison au préalable pour que ces noms ne vérifient pas le test de la lecture durative et / ou fréquentative. Les exemplifications ont parfois l'inconvénient de ne 'servir' que le but visé. Ainsi par exemple, il nous semple que les énoncés (39a), (40a) et (41a), présentés plus haut, portent essentiellement sur le versant massif des noms de qualité / sentiment / état. Du coup, l'adverbe beaucoup et / ou souvent accentue la grandeur dénotée 'en profondeur', ce qui va de pair avec l'impossibilité d'interpréter le verbe (aimer) et les adjectifs (gentil et fatigué) auxquels les noms en question sont apparentés sous la lecture durative (introduite par longtemps) ou fréquentative (introduite par souvent). Dans le cas contraire, c'est-à-dire lorsque nous entendons mettre en profil le versant comptable des noms, il est possible d'aboutir à une lecture, sinon durative, du moins fréquentative :

(55) Nom de sentiment

a. Paul a connu beaucoup d'amour dans sa vie

b. ? Paul a été souvent aimé

c. = Paul a été aimé plusieurs fois dans sa vie

(56) Nom de qualité

a. Nos familles nous ont prodigués beaucoup de gentillesse au fil des ans 
b. ? Nos familles ont été souvent gentilles avec nous

c. = Nos familles ont été plusieurs fois gentilles avec nous au fil des ans

(57) Nom d'état

a. Marie a ressenti beaucoup de fatigue durant sa dernière année de travail

b. ? Marie a été souvent fatiguée durant sa dernière année de travail

c. = Marie a été fatiguée plusieurs fois durant sa dernière année de travail

Nous soulignerons que la lecture fréquentative serait d'autant moins sujette à caution que nous nous aviserions de marquer les noms amour, gentillesse et fatigue au pluriel. Mais leur pluralisation les instancierait exclusivement dans l'espace temporel, ce qui reviendrait à ne mettre en profil que leur versant [+comptable]. Cette façon de faire aurait pour simple motif de prendre le contrepied de l'exemplification de Beauseroy, reprise sous (39-41). Les exemples (55a-57a) sont importants pour une tout autre raison. Le singulier des noms amour, gentillesse et fatigue a pour corollaire en effet de présupposer les deux interprétations, intensive (massive) et occurrentielle (comptable). Selon l'interprétation intensive, les grandeurs dénotées (amour, gentillesse et fatigue) sont conceptualisées comme intrinsèquement continues dans le cadre de périodes temporelles limitées. Les noms amour, gentillesse et fatigue occupent intensivement (en profondeur) des périodes limitées de temps, indiquées par les expressions dans sa vie, au fil des ans et sa derrière année de travail respectivement. Selon l'interprétation extensive, ces noms désignent des grandeurs intrinsèquement discontinues, consistant en un certain nombre d'épisodes et / ou d'expériences qui s'étendent sur les périodes indiquées. Le sens des expressions temporelles tient un rôle capital dans l'interprétation extensive des noms intensifs. Ces périodes doivent être assez longues pour favoriser la lecture fréquentative des noms intensifs. Ainsi, pour que les noms amours, gentillesse et fatigue reçoivent des interprétations fréquentatives, il faut que le cadre temporel de leur déroulement soit compatible avec la pluralité des épisodes accomplis.

On l'aura constaté, la complexité de la base conceptuelle des noms de qualité / sentiment / état justifie et sous-tend leurs interprétations à la fois sur la dimension [+massif] et sur la dimension [+comptable]. La capacité des noms intensifs à présupposer dans certains énoncés, comme (55-57), deux lectures sémantiquement distinctes, intensive (massive) et extensive (comptable), fournit la preuve que les traits sous-catégoriels [+massif] et [+comptable] certes s’opposent, mais ne s'excluent pas nécessairement. Nous en concluons que dans bon nombre de situations, la manifestation des noms sur le versant massif et / ou comptable ne correspond pas à des cas de recatégorisation, mais plutôt à des faits de polysémies lexicales, voire de polysémies lexicales systématiques.

\section{Conclusion}

Le phénomène de la recatégorisation demeure étroitement lié à la problématique des noms massifs et des noms comptables. Les nombreux passages entre les deux catégories conduit, dans une certaine mesure, à renoncer à une opposition massif / comptable radicale. Le phénomène tire son origine des données elles-mêmes de la langue. Toujours est-il que ces données sont assez complexes pour se suffire aux seuls recatégorisateurs décrits dans la littérature. C'est dans ce sens que nous avons tenté d'examiner un nouveau mode de recatégorisation, en l'occurrence le métonymiseur. Ce mode permet d'expliquer comment des noms basiquement [+massif] et / ou [+comptable] peuvent en usage métonymique apparaitre sous le trait inverse. Nous le trouvons à l'œuvre notamment dans les énoncés figuratifs. Il éclate en deux processus :

- l'abstractiveur massifiant rend compte du passage comptable $\rightarrow$ massif ;

- le concrétiseur individualisant rend compte du passage massif $\rightarrow$ comptable. 
L'intérêt de la postulation d'un mode de transfert de type métonymique - le métonymiseur ne tient pas seulement dans la description d'un type particulier de recatégorisation, mais plus particulièrement dans le rôle central que la métonymie remplit dans l'enrichissement de la structure informative des noms massifs et / ou comptables. En règle générale, la métonymie est considérée comme l'un des moteurs productifs de la polysémie lexicale : le phénomène d'extension de sens trouve souvent son fondement dans la conventionnalisation des emplois métonymiques.

La métonymie revêt une importance cruciale sur le chapitre de la distinction massif / comptable. Elle agit régulièrement sur la structure référentielle des noms massifs et / ou comptables et en modifie du coup les traits sous-catégoriels basiques. A titre de rappel, un nom intrinsèquement [+massif] et / ou [+comptable] peut par métonymie se manifester sous le trait sous-catégoriel de la catégorie opposée. Tant que cet emploi ne bénéficie pas de stabilité dans l'usage courant, il s'agit toujours d'une recatégorisation pragmatique puisque son interprétabilité est fonction du contexte global d'énonciation. Mais dès lors qu'il se conventionnalise dans l'usage, il constitue un fait de polysémie lexicale. Ce constat secoue notre conception classique de la distinction massif / comptable. Nous sommes ainsi conduit à reconsidérer la corrélation des traits sous-catégoriels [+massif] et [+comptable] sous de nouveaux rapports. En somme, dans la mesure où les traits [+massif] et [+comptable] peuvent, en termes de la polysémie lexicale, co-structurer les bases conceptuelles de certains noms, nous gagnerons toujours à les considérer sous un rapport d'opposition, mais non sous un rapport d'exclusion.

\section{Références}

Barner, D. et Snedeker, J. (2005). Quantity judgments and individuation: evidence that mass nouns count. Cognition, 97, 41-66.

Beauseroy, D. (2008). Les noms abstraits intensifs ou "noms statifs ": (non) projection du nombre et interprétation, in Durand J. Habert B., Laks B. (éds), Actes du CMLF 2008, 2389-2406.

Bloom, P. et Keleman, D. (1995). Syntactic cues in the acquisition of collective nouns. Cognition, 56, $1-30$.

Bunt, H. (1985). Mass terms and Model theoretic semantics. Cambridge: Cambridge University Press.

Cheng, L. et Sybesma, R. (1999). Bare and Not-So-Bare Nouns and the structure of NP. Linguistic Inquiry; 30, 509-542.

Chierchia, G. (1998). Reference to Kinds across Language. Natural Language Semantics, 6, 339-405.

Chierchia, G. (2010). Mass nouns, vagueness, and semantic variation. Synthese, 174, 99-149.

Goddard, C. (1994). Semantic Theory and Semantic Universals. In C. Goddard et A. Wierzbicka (éds.), Semantic and Lexical Universals - Theory and Empirical Findings, Philadelphia : John Benjamins, 7-29.

Goddard, C. (2009). A Piece of Cheese, A Grain of Sand: The Semantics of Mass Nouns and Unitizers. In J. Pelletier (éd.) Kinds, Things and Stuff, New York: Oxford UP, 132-165.

Goddard, C. et Wierzbicka, A. (2002). Meaning and Universal Grammar: Theory and Empirical Findings (Vol.1 et 2). Philadelphia : John Benjamins.

Galmiche, M. (1989). Massif/comptable : de l'un à l'autre et inversement. In J. David et G. Kleiber (éds.), Termes massifs et termes comptables, Paris : Klincksieck, 63-77.

Flaux, N. et Van de Velde, D. (2000). Les noms en français : esquisse de classement. Paris : Ophrys.

Herslund, M. (1998), Le français, langue à classificateurs ? In, A. Englebert et M. Pierrard (éds.), « La ligne claire » : de la linguistique à la grammaire. Mélanges offerts à M. Wilmet à l'occasion de son $60^{e}$ anniversaire. Paris : Duculot, 65-74. 
Imai, M. et Gentner, D. (1997). A cross-linguistic study of early word meaning: universal ontology and linguistic influence. Cognition, 62, 169-200.

Jackendoff, R. (1991). Parts and boundaries. Cognition, 41, 9-45.

Johnson, M. (1987). The Body in the Mind: The Bodily Basis of Meaning, Imagination and Reason. Chicago: Chicago University Press.

Kleiber, G. (1990). L’article LE générique. La généricité sur le mode massif. Genève : Librairie Droz.

Kleiber, G. (1994). Nominales. Essai de sémantique référentielle. Paris : Armand Colin.

Kleiber, G. (1999). Problèmes de Sémantique. La polysémie en question. Paris : Presses Universitaires du Septentrion.

Lakoff, G. (1987). Women, Fire and Dangerous Things: What Categories Reveal About the Mind. Chicago: University of Chicago Press.

Langacker, R.W. (1987). Foundations of Cognitive Grammar. Vol 1. Standford University Press.

Langacker, R.W. (1991). Foundations of Cognitive Grammar; Vol. 2. Descriptive Application. Standford University Press.

Langacker, R.W. (2013). Essentials of Cognitive Grammar. Oxford University Press.

Lemghari, E. (2010a), La base conceptuelle des noms abstraits. Vers la postulation du continuum massif $\leftrightarrow$ comptable, in Neuveu F., Muni Toke V., Durand J., Klingler T., Mondada L., Prevost S. (éds.), ), Actes du CMLF 2010, 1741- 1752.

Lemghari, E. (2010b). Complexité sous-catégorielle des noms massifs et comptables. Quelques faits de polysémie lexicale régulière. Revue de Sémantique et de Pragmatique, 28, 99-122.

Numberg, G. et Zaenen, A. (1997). La polysémie systématique dans la description lexicale. Langue française, 113, 12-23.

Obadia, M. (1976), (éds). Grammaire 3, Les Chemins de l'expression. Le lexique. Paris : Hachette.

Pelletier, F.J. (1975). Non-Singular reference : Some Preminaries. Philosophia, 5 , 4, 451-465.

Talmy, L. (2000). Toward a Cognitive Semantics, Vol. 1. Cambridge : MIT Press.

Van De Velde, D. (1995). Le spectre nominal. Des noms de matières aux noms d'abstractions. Paris : Société pour l'Information Grammaticale.

Vermote, T. (2014). Les noms de matériaux au crible de l’opposition massif-comptable. Une étude de corpus. Langue française, 183, 55-69.

Weinrich, U. (1966), Exploration in Semantic Theory, in T.A.Sebeok (éd.) Current Trends in Linguistics, Vol III, 395-477. Mouton : la Haye.

Wierzbicka, A. (1988). The Semantics of Grammar. Amsterdam : John Benjamin.

* Les remarques précieuses des relecteurs nous ont amené à une meilleure compréhension de la problématique étudiée. Nous les en remercions.

${ }^{1}$ L’interprétation la plus naturelle de cet énoncé ferait référence à la pluralité indéfinie des arbres chêne et peuplier dans l'endroit désigné.

2 R. Jackendoff (1991 : 24) rapporte (cf. note 11) que D. Lewis n’a jamais utilisé la notion de conditionneur universel dans ses écrits et qu'il l'attribue à son tour aux écrits d'un autre auteur dans les années soixante, Victor Yngve.

3 Un autre problème que soulève immanquablement la thèse de l'ellipse est en fait de ne pouvoir identifier l'entité conditionnante effacée indépendamment du contexte situationnelle spécifié. Une eau peut désigner aussi bien une bouteille qu'une caisse d'eau. Il en va de même pour un blanc/un 
rouge qui est susceptible de renvoyer, selon le contexte, tant à un verre qu'à une bouteille de blanc / de rouge. Le problème gagne en complexité avec ce dernier exemple ; en effet, si on admet qu'il y a là une ellipse, il va falloir alors déterminer avec exactitude ce qui se trouve effacé (verre, bouteille, caisse ou encore la substance vin elle-même), dans la mesure où l'expression un blanc / un rouge peut présupposer la co-occurrence de toutes ces entités conditionnantes : on dit naturellement un verre, une bouteille, une caisse de vin blanc / de vin rouge.

${ }^{4}$ Le défini la, neutre à la distinction massif / comptable, ne doit pas fausser notre jugement ; le SN la vengeance se laisse en effet facilement remplacer par le SN une vengeance. La différence de sens qui en résulte est celle-là même qui sous-tend la différence entre définitude et indéfinitude.

5 Les termes massifs et comptables sont utilisés ici au pluriel pour faire allusion aux efforts d'extension de la distinction massif / comptable à des paradigmes autres que le paradigme du nom commun, tels que le verbe, l'adjectif, le nom propre, la relative, etc.

${ }^{6}$ La coexistence paradoxale des traits massif et comptable dans la même structure référentielle globale de certains noms n'est point un fait idiosyncratique de la polysémie lexicale sur le mode de la distinction massif / comptable. Il existe en effet certains noms qui sont basiquement à la fois massif et comptable (pain, herbe, papier). Dans les prochaines décennies, les recherches ne s’intéresseraient que marginalement à la problématique de l'opposition massif / comptable. L'attention, sur ce chapitre, porterait, si n'est déjà entamé, sur le problème de distinguer entre trois faits : les faits de recatégorisation pragmatique, les faits de polysémies lexicales et les faits qui relèvent de l'hybridité de la sous-classe intermédiaire, c'est-à-dire la sous-classe des noms qui sont basiquement à la fois massif et comptable.

7 Une tentative de ce genre est trouvable dans (G. Kleiber, 1999). L'auteur constate que la thèse de G. Numberg et A. Zaenen (1997), qu'il appelle approche unitaire, a le défaut majeur de faire dépendre toutes les manifestations comptables des noms massifs d'un même principe de transfert général, en l'occurrence le broyeur. Il propose en contrepartie une approche non unitaire dont l'avantage essentiel est de montrer que certains emplois massifs de noms fondamentalement comptables ne correspondent pas à des cas de recatégorisation, mais à des cas de polysémies lexicales.

8 Le paradigme des noms d'instruments de musique n'est pas le seul paradigme qui manifeste une régularité sur le mode de la polysémie comptable $\rightarrow$ massif. Il en existe bien d'autres, tels que le paradigme des noms indiquant des scénarios d'activité, les noms de métier, etc. (cf. Lemghari (2010b), pour plus de détails).

9 C'est le cas, par exemple, de la matière or. L'usage ne fournit pas le pluriel ors par référence à des objets en or.

10 La sous-classe des noms d'états se compose de plusieurs types de noms dont les bases conceptuelles sont structurées par différents domaines cognitifs. Notre attention se limite ici aux noms d'états qui ont pour domaine cognitif primordial l'espace affectif. 